\title{
Alternative Trade-Offs in Data Envelopment Analysis: An Application to Hydropower Plants
}

\author{
Mohsen Mirzaei, ${ }^{1}$ Sohrab Kordrostami, ${ }^{1}$ Alireza Amirteimoori, ${ }^{2}$ and Mehrdad G. Chegini ${ }^{3}$ \\ ${ }^{1}$ Department of Applied Mathematics, Islamic Azad University, Lahijan, Iran \\ ${ }^{2}$ Department of Applied Mathematics, Islamic Azad University, Rasht, Iran \\ ${ }^{3}$ Department of Management, Islamic Azad University, Rasht, Iran
}

Correspondence should be addressed to Alireza Amirteimoori; aamirteimoori@gmail.com

Received 27 September 2015; Accepted 6 December 2015

Academic Editor: Fazal M. Mahomed

Copyright (C) 2016 Mohsen Mirzaei et al. This is an open access article distributed under the Creative Commons Attribution License, which permits unrestricted use, distribution, and reproduction in any medium, provided the original work is properly cited.

\begin{abstract}
In multidimensional input/output space, the behavior of the firms can be analyzed by using efficient frontier or supporting surfaces of production technology. To this end, mathematicians are interested to use marginal rates of substitutions. The piecewise linear frontier of data envelopment analysis (DEA) technology is not differentiable at the extreme points and marginal rates calculation is valid only for small changes in one or more variables. The existing trade-off analysis methods calculate the maximum changes in a specific throughput when another throughput is changed. We will show that binding efficient supporting surfaces of an efficient point may be used to define different marginal rates of substitutions and in this sense, we get different marginal rates to each frontier point.
\end{abstract}

\section{Introduction}

Data envelopment analysis (DEA) is a LP-based nonparametric technique for measuring the relative performances of firms that use multiple inputs to produce multiple outputs. The literature on the performance measurement using DEA has grown substantially since Charnes et al. [1] introduced the traditional CCR model based on the maximum radial reduction in inputs. It has been widely used in performance measurement of many business and industry applications. A complete literature and surveys can be found in Cooper et al. [2] and Cook and Seiford [3].

While much DEA-based research has been directed to the application of DEA for performance measurement, fewer works have been made on its properties as a production function model. Production technology in DEA is extrapolated with the observed inputs and outputs and the boundary points of this set construct the efficient frontier or supporting hyperplanes of the technology. Supporting hyperplanes of production technology enable us to analyze the relation between two different throughputs. Mathematicians are very interested to work with efficient frontiers or supporting surfaces of production possibility set to analyze the behavior of firms in the multidimensional input/output space.

The knowledge of trade-offs in a production process is an important subject to the managers. For instance, managers are interested to know the additional amount of a certain input that is required in order to increase a particular output by a small fixed amount.

Huang et al. [4] proposed a general method for calculating the rates of change of outputs to the inputs along efficient surface of DEA production set. Rosen et al. [5] directly studied the problem of marginal rate of substation on efficient frontier and presented a general framework for the calculation of trade-offs between two variables in DEA. Cooper et al. [6] modified the classic additive DEA model and from the optimal slack values of this model they derived the marginal rates and elasticities of substitution.

Krivonozhko et al. [7] have used the supporting hyperplanes and efficient surfaces to calculate marginal rates of substitution in DEA. In the meantime, using supporting hyperplanes of production technology, Førsund and Hjalmarsson [8] have calculated scale elasticity in DEA models. Førsund et al. [9] have also proposed two ways of 
obtaining numerical values of scale elasticity by directindirect approaches and then they compared the two approaches by real data. Balk et al. [10] generalized the concept of scale elasticity to accommodate changes in any given direction in input-output space. Khoshandam et al. [11] proposed a production in which a group of variables are changed in a given direction and the effect of this change on some throughput is calculated. In their second paper, Khoshandam et al. [11] studied the problem of marginal rates of substitution in the presence of nondiscretionary factors.

The abovementioned approaches calculate a single measure of marginal rates of substitution and these trade-off analysis methods are given in optimistic case in the sense that they calculate the maximum changes in a specific throughput when another throughput is changed by a small quantity. In this sense, the new frontier point may be very far from the original frontier point. However we can equivalently look for a piece of the frontier in which the new frontier point is closing as much as possible to the original point.

We show that binding supporting surfaces of production set in an efficient point can be used to define marginal rates of substitutions and hence we may calculate different marginal rates in efficient points. We show this geometrically by a simple example.

The rest of the paper is organized as follows: marginal rates substitutions are introduced in the next section. Section 3 introduces two marginal rates in optimistic and pessimistic cases. Marginal rates in the presence of undesirable outputs and in the absence of explicit inputs are given in Section 4. A real application is used to illustrate the proposed approach. The paper ends with concluding remarks.

\section{Marginal Rates of Substitution}

In mathematical economics, marginal rate of substitution is employed to calculate the relative marginal utility. Mathematicians are often interested to work with efficient surfaces, supporting hyperplanes and Pareto-efficient surfaces. In multidimensional input-output space, the behavior of a production unit can easily be read using the supporting hyperplanes of the DEA production possibility set without any loss of mathematical rigor. In this section, we briefly introduce the marginal rate of substitution. Consider a general process in which an output vector $y=\left(y_{1}, \ldots, y_{s}\right) \in \mathrm{R}_{+}{ }_{+}$is produced by consuming the input vector $x=\left(x_{1}, \ldots, x_{m}\right) \in \mathrm{R}^{m}{ }_{+}$. There are a set of $n \mathrm{DMU}_{j}: j=1, \ldots, n$, each of which is characterized by a throughput vector $z_{j}=\left(x_{j}, y_{j}\right)^{t}$ in which $x_{j}=$ $\left(x_{1 j}, \ldots, x_{m j}\right)$ and $y_{j}=\left(y_{1 j}, \ldots, y_{s j}\right)$ are nonzero and nonnegative vectors. To simplify, the throughput matrix is shown as follows:

$$
\begin{aligned}
z & =\left(z_{1}, \ldots, z_{n}\right) \\
& =\left(\begin{array}{ccccc}
z_{11} & \ldots & z_{1 j} & \ldots & z_{1 n} \\
\vdots & & \vdots & & \vdots \\
z_{i 1} & \ldots & z_{i j} & \ldots & z_{\text {in }} \\
\vdots & & \vdots & & \vdots \\
z_{(m+s), 1} & \ldots & z_{(m+s), j} & \ldots & z_{(m+s), n}
\end{array}\right) .
\end{aligned}
$$

Let $F(x, y)=0$ be the boundary of the production technology and without loss of generality, we assume that

$$
\begin{gathered}
\frac{\partial F(x, y)}{\partial x_{i}}<0 \quad i=1, \ldots, m \\
\frac{\partial F(x, y)}{\partial y_{r}}>0 \quad r=1, \ldots, s .
\end{gathered}
$$

Moreover, we assume that the production technology shows free disposability or in other words $F(x, y)$ is assumed to be continuously differentiable. Let $z_{o}=\left(x_{o}, y_{o}\right)$ be a frontier point; that is, $F\left(z_{o}\right)=F\left(x_{o}, y_{o}\right)=0$.

Definition 1. The marginal rate of substitution of the $j$ th throughput to the $k$ th throughput at the frontier point $z_{o}$ is defined as follows:

$$
\begin{aligned}
\operatorname{MR}_{j k}^{+}\left(z_{o}\right) & =\left(\frac{\partial z_{j o}}{\partial z_{k o}}\right)_{Z_{o}^{+}} \\
& =\lim _{h \rightarrow 0^{+}} \frac{f_{j}\left(z_{1 o}, \ldots, z_{k o}+h, \ldots, z_{m+s o}\right)}{h}, \\
\operatorname{MR}_{j k}^{-}\left(z_{o}\right) & =\left(\frac{\partial z_{j o}}{\partial z_{k o}}\right)_{Z_{o}^{-}} \\
& =\lim _{h \rightarrow 0^{-}} \frac{f_{j}\left(z_{1 o}, \ldots, z_{k o}+h, \ldots, z_{m+s o}\right)}{h} .
\end{aligned}
$$

As we know, in real applications, $F(x, y)$ is not known exactly and hence we instead use the empirical production technologies such as DEA production possibility set. This set is structured axiomatically and the boundary points of this set construct the empirical production function that is a piecewise linear function. Due to the nature of the DEA efficient frontier, marginal rates are not uniquely defined in the extreme efficient units on the frontier and hence, we should calculate the marginal rate of substitution to right and left.

A DEA-based procedure to calculate the marginal rates of substitution of throughput $j$ to throughput $k$ from right at the frontier point $z_{o}=\left(x_{o}, y_{o}\right)$ is given by Asmild et al. [12] as follows:

$$
\operatorname{MR}_{j k}^{+}\left(z_{o}\right)=\frac{z_{j o}^{*}-z_{j o}}{h},
$$

in which $h$ is a small positive number and $z_{j o}^{*}$ is the optimal solution to the following LP problem:

$$
\begin{array}{ll}
\operatorname{Max} & Z_{j o}^{*} \\
\text { s.t. } & \sum_{t=1}^{n} \lambda_{t} z_{l t} \geq z_{l o} \quad l \neq j, k \\
& \sum_{t=1}^{n} \lambda_{t} z_{j t} \geq z_{j o}^{*} \\
& \sum_{t=1}^{n} \lambda_{t} z_{k t} \geq z_{k o}+h
\end{array}
$$


TABLE 1: The data set for simple example.

\begin{tabular}{cccccccc}
\hline & $\mathrm{A}$ & $\mathrm{B}$ & $\mathrm{C}$ & $\mathrm{D}$ & $\mathrm{E}$ & $\mathrm{F}$ & $\mathrm{G}$ \\
\hline$x_{1}$ & 0.9 & 0.5 & 1.1 & 0.2 & 2.2 & 2.8 & 3 \\
$x_{2}$ & 1.63 & 1.36 & 1.55 & 2.15 & 2.04 & 1.40 & 2.04 \\
$y_{1}$ & 0.65 & 0.35 & 0.65 & 0.55 & 1.2 & 0.8 & 1.3 \\
\hline
\end{tabular}

$$
\begin{aligned}
& \sum_{t=1}^{n} \lambda_{t}=1 \\
& \lambda_{t} \geq 0 .
\end{aligned}
$$

Substituting $-h$ instead of $h$ in the foregoing procedure gives the left marginal rates of substitution.

In this procedure, the new frontier point $z_{o}^{\prime}=\left(z_{1 o}, \ldots\right.$, $\left.z_{k o}+h, \ldots, z_{j o}^{*}, \ldots, z_{m+s o}\right)$ is calculated in optimistic case in the sense that $z_{j o}^{*}$ is maximized. There may be another point on the frontier and we can use this point instead of $z_{j o}^{*}$. In the next section, two different marginal rates of substitutions are calculated, one in optimistic case and another one in pessimistic case.

\section{Different Marginal Rates}

As we stated in the previous section, the classic trade-off analysis is given in the optimistic case and hence they determine the maximum changes in a specific component of the throughput vector when a specific component is increased (or decreased) by a small quantity.

Generally, we solve the following LP problem to calculate the marginal rates of substitution:

$$
\begin{array}{ll}
\operatorname{Max} & z_{k o}^{\prime} \\
\text { s.t. } & z_{o}^{\prime} \in T,
\end{array}
$$

in which $z_{o}^{\prime}=\left(z_{1 o}, \ldots, z_{k o}+h, \ldots, z_{m+s o}\right)$ is the new input/ output vector after changes. The maximization objective function in the LP problem (6) is given in optimistic case and hence, it finds the maximum value to a specific throughput when another throughput is changed by a small quantity. In model (6), we look for a piece of the frontier in which the specified component is maximized. We can equivalently look for a piece of the frontier in which the component is minimized. Needless to say in both cases the new point is a frontier point and this is what we want.

We show this by the following simple example. We use a simple example with seven DMUs with two inputs $x_{1}$ and $x_{2}$ and one output $y_{1}$. The data are summarized in Table 1 .

The BCC model of Banker et al. [13] has been used and we saw that all DMUs are efficient. The production technology in variable returns to scale case is depicted in Figure 1.

Consider $\mathrm{DMU}_{\mathrm{A}}$ with $z_{1}:\left(-x_{11},-x_{21}, y_{1}\right)=(-0.9,-1.63$, $0.65)$. Suppose that we change $y_{1}$ to $y_{1}+h$ and the response of this change on $x_{1}$ and $x_{2}$ is needed. As the figure shows, in $\mathrm{DMU}_{\mathrm{A}}$, four efficient facets, $\mathrm{ABC}, \mathrm{ABD}, \mathrm{ADE}$, and $\mathrm{ACE}$,

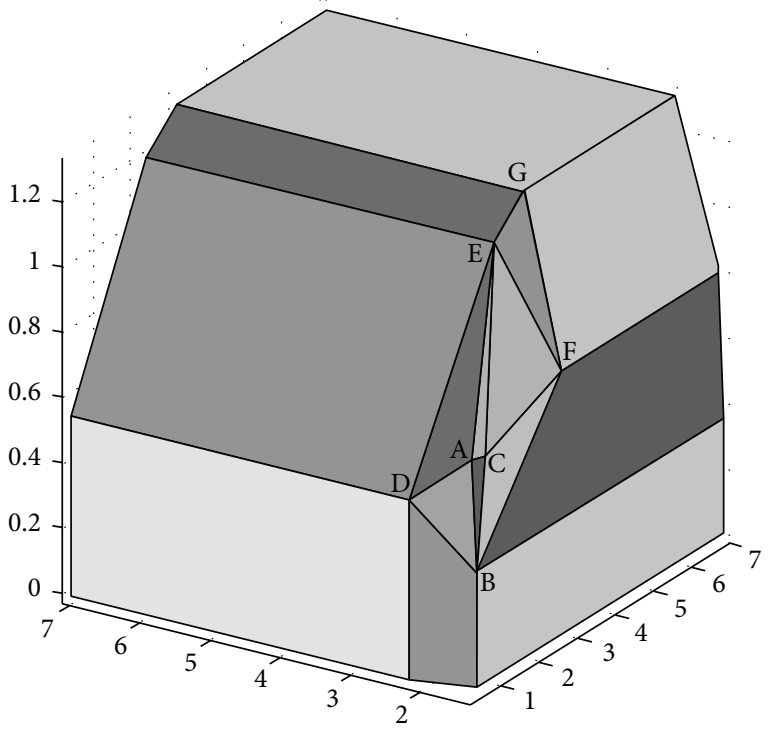

FIGURE 1: The production technology.

are binding and the optimistic approach finds a new point on facet ACE with $\left(-x_{11},-x_{21}, y_{1}^{*}\right)=(-1.1364,-1.7045,0.75)$ and $\mathrm{MR}_{13}^{(+)}=2.364$ and $\mathrm{MR}_{23}^{(+)}=0.745 . \mathrm{MR}_{13}^{(+)}$is the value of change in first input that results when the single output is increased by 0.1 units. Note that $\left(-x_{11},-x_{21}, y_{1}^{*}\right)=(-1.1364$, $-1.7045,0.75)$ is the result of the following model:

$$
\begin{array}{ll}
\text { Min } \quad z_{1}^{*}+z_{2}^{*} \\
\text { s.t. } \quad-0.9 \lambda_{1}-0.5 \lambda_{2}-1.1 \lambda_{3}-0.2 \lambda_{4}-2.2 \lambda_{5} \\
\quad-2.8 \lambda_{6}-3 \lambda_{7} \geq-z_{1}^{*} \\
-1.63 \lambda_{1}-1.36 \lambda_{2}-1.55 \lambda_{3}-2.15 \lambda_{4}-2.04 \lambda_{5} \\
\quad-1.4 \lambda_{6}-2.04 \lambda_{7} \geq-z_{2}^{*} \\
0.65 \lambda_{1}-0.35 \lambda_{2}-0.65 \lambda_{3}-0.55 \lambda_{4}-1.2 \lambda_{5} \\
\quad-0.8 \lambda_{6}-1.3 \lambda_{7} \geq 0.65+0.1 \\
\lambda_{1}+\lambda_{2}+\lambda_{3}+\lambda_{4}+\lambda_{5}+\lambda_{6}+\lambda_{7}=1 \\
\lambda_{1}, \lambda_{2}, \lambda_{3}, \lambda_{4}, \lambda_{5}, \lambda_{6}, \lambda_{7} \geq 0 .
\end{array}
$$

However, all points on the facets $\mathrm{ABC}, \mathrm{ABD}$, and $\mathrm{ADE}$ can provide us a frontier point to calculate a new marginal rate. If we change the direction of movement, a new point on another frontier is obtained. For example, point $\left(-x_{11},-x_{21}, y_{1}^{*}\right)=$ $(-0.8154,-2.1162,0.75)$ is a frontier point on the facet ADE with $\mathrm{MR}_{13}^{(-)}=-0.8462$ and $\mathrm{MR}_{23}^{(-)}=-4.8615$. Let us compare these two rates: In $\mathrm{DMU}_{\mathrm{A}}, \mathrm{MR}_{13}^{(+)}$and $\mathrm{MR}_{23}^{(+)}$mean that if we increase $y_{1}$ from 0.65 to 0.75 , we will remain on the efficient frontier if the two inputs are, respectively, changed to 1.1364 and 1.7045 , while $\mathrm{MR}_{13}^{(-)}$and $\mathrm{MR}_{23}^{(-)}$mean that, with $h=+0.1$, we will remain on the efficient frontier if the two inputs are, respectively, changed to 0.8154 and 2.1162 . It is interesting; there is a substantial gap between the two new points on two different efficient facets. Note that 
$\left(-x_{11},-x_{21}, y_{1}^{*}\right)=(-0.8154,-2.1162,0.75)$ is the optimal solution of the following LP problem:

$$
\begin{array}{ll}
\text { Min } \quad-z_{1}^{*}+z_{2}^{*} \\
\text { s.t. } \quad-0.9 \lambda_{1}-0.5 \lambda_{2}-1.1 \lambda_{3}-0.2 \lambda_{4}-2.2 \lambda_{5} \\
\quad-2.8 \lambda_{6}-3 \lambda_{7} \geq-z_{1}^{*} \\
-1.63 \lambda_{1}-1.36 \lambda_{2}-1.55 \lambda_{3}-2.15 \lambda_{4}-2.04 \lambda_{5} \\
\quad-1.4 \lambda_{6}-2.04 \lambda_{7} \geq-z_{2}^{*} \\
0.65 \lambda_{1}-0.35 \lambda_{2}-0.65 \lambda_{3}-0.55 \lambda_{4}-1.2 \lambda_{5} \\
\quad-0.8 \lambda_{6}-1.3 \lambda_{7} \geq 0.65+0.1 \\
\lambda_{1}+\lambda_{2}+\lambda_{3}+\lambda_{4}+\lambda_{5}+\lambda_{6}+\lambda_{7}=1 \\
\lambda_{1}, \lambda_{2}, \lambda_{3}, \lambda_{4}, \lambda_{5}, \lambda_{6}, \lambda_{7} \geq 0 .
\end{array}
$$

As a result, we have calculated two marginal rates: $\left(\mathrm{MR}_{13}^{(+)}\right.$, $\left.\mathrm{MR}_{23}^{(+)}\right)$and $\left(\mathrm{MR}_{13}^{(-)}, \mathrm{MR}_{23}^{(-)}\right)$.

We can summarize the foregoing procedure as follows.

To calculate the two marginal rates of substitutions of throughputs in group $M$ to throughputs in $N$, at the frontier point $z_{o}=\left(-x_{o}, y_{o}\right)$, we can use the following three-step procedure:

(i) Choose a small number $h$ for throughput $k$.

(ii) Solve the following two LP problems $\left(d_{l}^{(1)}\right.$ and $d_{l}^{(2)}$ are user-defined coefficients):

$$
\begin{aligned}
z_{o}^{(+)}=\operatorname{Max} & \sum_{l \in M} d_{l}^{(1)} z_{l o}^{*} \\
\text { s.t. } \quad & \sum_{t=1}^{n} \lambda_{t} z_{t l} \geq z_{l o}^{*}, \quad l=1,2, \ldots, m+s, \\
& \sum_{t=1}^{n} \lambda_{t}=1, \\
& z_{l o}^{*}=z_{l o}, \quad l \notin M, \quad l \notin N, \\
& z_{k o}^{*}=z_{k o}+h, \quad l \in N, \\
& \lambda_{t}, z_{l o}^{*} \geq 0, \quad \forall t, l, \\
& \sum_{l \in M} d_{l}^{(2)} z_{l o}^{*} \\
z_{o}^{(-)}=\operatorname{Max} & \sum_{t=1}^{n} \lambda_{t} z_{t l} \geq z_{l o}^{*}, \quad l=1,2, \ldots, m+s, \\
& \sum_{t=1}^{n} \lambda_{t}=1, \\
& z_{l o}^{*}=z_{l o}^{*}, \quad l \notin M, \quad l \notin N, \quad \forall t, l . \\
& z_{k o}^{*}=z_{k o}+h, \quad l \in N, \\
&
\end{aligned}
$$

(iii) Calculate the optimistic and pessimistic marginal rates of substitutions from right as follows:

$$
\begin{aligned}
\operatorname{MR}_{j k}^{(+)}\left(z_{o}\right)=\frac{z_{j o}^{(+)}-z_{j o}}{h}, & \\
\operatorname{MR}_{j k}^{(-)}\left(z_{o}\right)=\frac{z_{j o}^{(-)}-z_{j o}}{h} & \\
& \forall j \in M, k \in N .
\end{aligned}
$$

Note that both marginal rates of substitutions from left can be calculated by replacing $-h$ instead of $h$. It is easy to show that both projection points obtained from models (9) and (10) are frontier points. Moreover, different weight vectors $d$ lead to different marginal rates on different efficient facets. The weight vector $d$ is a user-defined vector that determines the direction of moving to efficient facet.

\section{Marginal Rates in the Presence of Bad Outputs}

Now suppose we face a production system without explicit inputs and the system has just desirable and undesirable outputs (indeed, in such a system, inputs consumption is not important to the decision makers). Such a system may have considerable applications in real life when the process has focused on outputs production. In what follows, we reformulate the procedure to production systems without explicit inputs in the presence of both, desirable and undesirable outputs.

Suppose we have $n \mathrm{DMU}_{j}: j=1, \ldots, n$, with the good output vector $y_{j}=\left(y_{1 j}, \ldots, y_{s j}\right) \geq 0$ and bad output vector $z_{j}=\left(z_{1 j}, \ldots, z_{p j}\right) \geq 0$. Masoumzadeh et al. [14] have used the weak disposability assumption of Shephard [15] to propose the following linear production technology to handle undesirable outputs in production system without explicit inputs:

$$
\begin{aligned}
T_{\mathrm{WI}} & =\left\{(y, z): \sum_{j=1}^{n} \lambda_{j} y_{j} \geq y, \sum_{j=1}^{n} \lambda_{j} z_{j}=z, \sum_{j=1}^{n} \lambda_{j}=\theta, 0\right. \\
& \left.\leq \theta \leq 1, \lambda_{j} \geq 0, \forall j\right\} .
\end{aligned}
$$

If we want to evaluate firm " $o$ " in terms of the abatement potential in bad outputs, we can use the following linear programming problem:

$$
\begin{array}{ll}
\text { Min } & \rho \\
\text { s.t. } & \sum_{j=1}^{n} \lambda_{j} y_{r j} \geq y_{r o}, \quad r=1,2, \ldots, s, \\
& \sum_{j=1}^{n} \lambda_{j} z_{p j} \geq \rho z_{p o}, \quad p=1,2, \ldots, P, \\
& \sum_{j=1}^{n} \lambda_{j}=\theta, \\
& 0 \leq \theta \leq 1, \quad \lambda_{j} \geq 0, \quad j=1,2, \ldots, n .
\end{array}
$$


Now, we reformulate the foregoing three-step procedure to calculate the marginal rates of substitution of the undesirable outputs in $N$ to the desirable outputs in $M$ from right:

(i) Choose a small number $h$.

(ii) Solve the following two LP problems:

$$
\begin{array}{ll}
\operatorname{Max} & \sum_{p \in M} d_{p}^{(1)} z_{p}^{(+)} \\
\text {s.t. } & \sum_{j=1}^{n} \lambda_{j} y_{r j} \geq y_{r o}, \quad r=1,2, \ldots, s, r \notin N, \\
& \sum_{j=1}^{n} \lambda_{j} y_{r j} \geq y_{r o}+h, \quad r \in N, \\
& \sum_{j=1}^{n} \lambda_{j} z_{p j} \geq z_{p o}, \quad p=1,2, \ldots, P, p \notin M, \\
& \sum_{j=1}^{n} \lambda_{j} z_{p j} \geq z_{p}^{(+)}, \quad p \in M, \\
& \sum_{j=1}^{n} \lambda_{j}=\theta, \\
& 0 \leq \theta \leq 1, \lambda_{j}, z_{p}^{(+)} \geq 0, \quad \forall j, p,
\end{array}
$$

$\operatorname{Max} \sum_{p \in M} d_{p}^{(2)} z_{p}^{(-)}$

s.t. $\quad \sum_{j=1}^{n} \lambda_{j} y_{r j} \geq y_{r o}, \quad r=1,2, \ldots, s, r \notin N$,

$$
\begin{aligned}
& \sum_{j=1}^{n} \lambda_{j} y_{r j} \geq y_{r o}+h, \quad r \in N, \\
& \sum_{j=1}^{n} \lambda_{j} z_{p j} \geq z_{p o}, \quad p=1,2, \ldots, P, p \notin M,
\end{aligned}
$$$$
\sum_{j=1}^{n} \lambda_{j} z_{p j} \geq z_{p}^{(-)}, \quad p \in M
$$$$
\sum_{j=1}^{n} \lambda_{j}=\theta
$$$$
0 \leq \theta \leq 1, \lambda_{j}, z_{p}^{(-)} \geq 0, \quad \forall j, p .
$$

(iii) Calculate the optimistic and pessimistic marginal rates of substitutions from right as follows:

$$
\begin{aligned}
& \operatorname{MR}_{p k}^{(+)}\left(y_{o}, z_{o}\right)=\frac{z_{p}^{(+)}-z_{p o}}{h}, \\
& \operatorname{MR}_{p k}^{(-)}\left(y_{o}, z_{o}\right)=\frac{z_{p}^{(-)}-z_{p o}}{h},
\end{aligned}
$$

$$
\forall p \in M, k \in N \text {. }
$$

Again, both marginal rates of substitutions from left can be calculated by replacing $-h$ instead of $h$.

\section{Real World Application}

To illustrate the marginal rates of substitution in the real cases, consider the following example that uses data describing the quality of the services provided by the largest hydropower plants operating in Brazil. The data has been taken from Zanella et al. [16]. These plants are under the performance monitoring of the Brazilian Independent System Operator (ISO) with capacity above 100-megawatt (MW) electricity. The data consist of 81 hydropower plants in Brazil. As Zanella et al. [16] stated, around 90 percent of the electrical energy of Brazil is supplied by hydropower plants and these 81 plants cover $82 \%$ of the Brazilian total installed capacity. In this sense, this selected sample is a good representation of the power supply in Brazil.

The data set consist of three different indicators as outputs that have been provided by Brazilian Independent System Operator. Of these three indicators, two indicators are considered as undesirable outputs and one is the single desirable output.

Now, we introduce these three indicators: The proportion of time that the plant is available or in operation is considered as the single desirable output (availability factor $\left(y_{1}\right)$ ). The first undesirable output is the average time to repair $\left(z_{1}\right)$ which accounts for the number of hours unavailable to operate and under forced maintenance per forced outages. Finally, the failure rate $\left(z_{2}\right)$ is considered as the second undesirable output. This indicator shows the number of failures occurring per hour of operation. Model (13) has been used in this data set and we saw that ten plants are efficient (plants \#4,

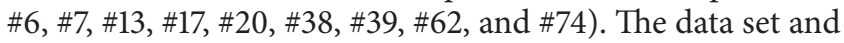
results are listed in Table 2 . Columns five and ten show the relative efficiencies obtained from our proposed model (13).

Suppose that $M=\{1,2\}$ and $N=\{1\}$ and we want to calculate the response of changes in the undesirable outputs $z_{1}$ and $z_{2}$ to the change in the desirable output $y$. It is important to note that both $h=0.5$ and $h=-0.5$ yield feasible points for all efficient plants. This means that increasing or decreasing 0.5 units in the desirable output leads to a point inside of the production technology. The results for two different values of $h$ are given in Tables 3 and 4 . The first two columns of Tables 3 and 4 show the new values of $z_{1}$ and $z_{2}$. The marginal rates of substitutions are given in the last two columns.

Now, we analyze the results for three sample plants, plants 7,13 , and 17. As the last two columns of Table 3 show, of ten plants, nine plants confront reduction in the bad outputs if we increase the single desirable output by 0.5 units.

First consider plant 13: If we increase the availability factor from its current value 95.64 to $96.14(h=0.5)$, with $d=(1,1)$, the average time to repair $\left(z_{1}\right)$ and the failure rate $\left(z_{2}\right)$ are, respectively, decreased to 0.1293 and 0.2568 with $\mathrm{MR}_{11}^{(+)}=$ -1.9414 and $\mathrm{MR}_{12}^{(+)}=-0.0028$. In plant 17 , if we increase 0.5 units in the availability factor, both undesirable factors $z_{1}$ and $z_{2}$ are increased to 0.1307 and 0.2613 , respectively. The marginal rates in this case are $\mathrm{MR}_{11}^{(+)}=0.0013$ and 
TABLE 2: The data set and results.

\begin{tabular}{|c|c|c|c|c|c|c|c|c|c|}
\hline $\begin{array}{l}\text { Plant } \\
J\end{array}$ & $y_{1}$ & $z_{1}$ & $z_{2}$ & Efficiency & $\begin{array}{c}\text { Plant } \\
J\end{array}$ & $y_{1}$ & $z_{1}$ & $z_{2}$ & Efficiency \\
\hline U1 & 93.62 & 165.56 & 4.25 & 0.0699 & U41 & 92.8 & 6.38 & 7.55 & 0.0331 \\
\hline $\mathrm{U} 2$ & 56.97 & 24.71 & 3.09 & 0.0509 & U42 & 92.22 & 4.88 & 5.01 & 0.0496 \\
\hline U3 & 90.28 & 15.11 & 1.3 & 0.1947 & U43 & 97.9 & 6.39 & 10.82 & 0.347 \\
\hline $\mathrm{U} 4$ & 98.46 & 9.94 & 2.35 & 1 & $\mathrm{U} 44$ & 92.95 & 31.83 & 4.74 & 0.0539 \\
\hline U5 & 93.45 & 15.26 & 2.83 & 0.0902 & U45 & 73.52 & 12.77 & 3.32 & 0.0601 \\
\hline U6 & 98.6 & 14.92 & 1.58 & 1 & U46 & 96.19 & 24.23 & 2.86 & 0.0933 \\
\hline U7 & 86.98 & 314.69 & 1.51 & 1 & U47 & 95.21 & 5.76 & 5.78 & 0.0444 \\
\hline U8 & 89.23 & 273.47 & 1.86 & 0.318 & U48 & 92.34 & 8.15 & 0.89 & 0.2879 \\
\hline U9 & 94.39 & 3.72 & 2.44 & 0.1044 & U49 & 65.99 & 79.01 & 3.07 & 0.0641 \\
\hline U10 & 93.66 & 38.9 & 1.5 & 0.1865 & U50 & 85.54 & 1.15 & 12.19 & 0.122 \\
\hline U11 & 96.74 & 0.72 & 1.24 & 0.3203 & U51 & 96.48 & 5.13 & 6.55 & 0.0397 \\
\hline U12 & 95.16 & 136.68 & 2.66 & 0.1208 & U52 & 73.89 & 6.17 & 3.7 & 0.0539 \\
\hline U13 & 95.64 & 1.1 & 0.26 & 1 & U53 & 95.72 & 0.58 & 2.07 & 0.2295 \\
\hline U14 & 97.18 & 20.31 & 0.57 & 0.9174 & U54 & 97.61 & 24.21 & 3.61 & 0.2453 \\
\hline U15 & 90.85 & 5.09 & 15.16 & 0.0245 & U55 & 94.79 & 3.03 & 14.11 & 0.0446 \\
\hline U16 & 94.29 & 5.36 & 2.44 & 0.1044 & U56 & 93.1 & 21.82 & 9.86 & 0.0255 \\
\hline U17 & 96.66 & 0.13 & 0.26 & 1 & U57 & 78.82 & 74.25 & 5.86 & 0.0379 \\
\hline U18 & 79.25 & 574.77 & 4.51 & 0.0973 & U58 & 97.83 & 11.87 & 6.31 & 0.293 \\
\hline U19 & 92.44 & 19.31 & 3.15 & 0.0804 & U59 & 92.49 & 92.49 & 8 & 0.0324 \\
\hline U20 & 97.69 & 2.79 & 1.19 & 1 & U60 & 91.65 & 3.87 & 1.93 & 0.1283 \\
\hline $\mathrm{U} 21$ & 95.04 & 13.59 & 2.78 & 0.0932 & U61 & 91.87 & 14.14 & 3.44 & 0.0726 \\
\hline U22 & 84.07 & 26.03 & 14.72 & 0.0154 & U62 & 98.17 & 0.33 & 10.11 & 1 \\
\hline U23 & 92.95 & 7.15 & 10.82 & 0.0231 & U63 & 96.96 & 5.47 & 4.08 & 0.146 \\
\hline U24 & 75.23 & 43.27 & 9.83 & 0.0208 & U64 & 93.38 & 6.99 & 1.53 & 0.1662 \\
\hline U25 & 97.56 & 6.94 & 5.44 & 0.2658 & U65 & 93.87 & 2.85 & 0.39 & 0.6628 \\
\hline U26 & 93.08 & 0.97 & 15.58 & 0.183 & U66 & 88.28 & 4.83 & 10.46 & 0.0247 \\
\hline U27 & 94.58 & 5.56 & 1.81 & 0.1416 & U67 & 78.45 & 24.45 & 4.12 & 0.0521 \\
\hline U28 & 96.79 & 5.57 & 6.86 & 0.0645 & U68 & 95.57 & 50.45 & 4.94 & 0.0539 \\
\hline U29 & 95.77 & 4.7 & 1.07 & 0.2436 & U69 & 93.98 & 38.87 & 21.63 & 0.0117 \\
\hline U30 & 94.42 & 17.88 & 0.9 & 0.3049 & U70 & 98.2 & 6.57 & 4.12 & 0.8721 \\
\hline U31 & 90.96 & 30.29 & 6.45 & 0.0384 & U71 & 96.98 & 34.69 & 8.86 & 0.0546 \\
\hline U32 & 96.87 & 2.33 & 3.38 & 0.1792 & U72 & 96.74 & 2.22 & 13.19 & 0.0639 \\
\hline U33 & 90.88 & 1.83 & 7.89 & 0.0702 & U73 & 93.97 & 6.61 & 21.4 & 0.0196 \\
\hline U34 & 91.04 & 143.22 & 2.9 & 0.1049 & U74 & 97.64 & 0.32 & 2.58 & 1 \\
\hline U35 & 97.01 & 3.86 & 8.1 & 0.1156 & U75 & 84.68 & 8.28 & 3.68 & 0.0622 \\
\hline U36 & 85.86 & 14.29 & 19.88 & 0.0116 & U76 & 72.69 & 116.59 & 10.11 & 0.0202 \\
\hline U37 & 96.21 & 0.93 & 1.37 & 0.189 & U77 & 95.86 & 11.94 & 2.6 & 0.1005 \\
\hline U38 & 98.4 & 20.24 & 0.75 & 1 & U78 & 94.62 & 11.25 & 2.36 & 0.1092 \\
\hline U39 & 98.45 & 7.05 & 5.15 & 1 & U79 & 94.81 & 23.17 & 2.06 & 0.1289 \\
\hline \multirow[t]{2}{*}{$\mathrm{U} 40$} & 73.91 & 4.21 & 1.49 & 0.1343 & U80 & 97.61 & 1.01 & 10.01 & 0.3034 \\
\hline & & & & & U81 & 80.03 & 3.23 & 7.07 & 0.0335 \\
\hline
\end{tabular}


TABLE 3: Results with $h=0.5$ and $d=(1,1)$.

\begin{tabular}{lcccc}
\hline & $z_{1}^{(+)}$ & $z_{2}^{(+)}$ & $\mathrm{MR}_{11}^{(+)}$ & $\mathrm{MR}_{12}^{(+)}$ \\
\hline U4 & 0.13313 & 0.2662 & -19.6138 & -4.1676 \\
U6 & 0.1333 & 0.2666 & -29.5734 & -2.6269 \\
U7 & 0.1177 & 0.2353 & -629.145 & -2.5494 \\
U13 & 0.1293 & 0.2586 & -1.9414 & -0.0028 \\
U17 & 0.1307 & 0.2613 & 0.0013 & 0.0027 \\
U20 & 0.1321 & 0.26411 & -5.3159 & -1.8518 \\
U38 & 0.1330 & 0.2660 & -40.2140 & -0.9680 \\
U39 & 0.1330 & 0.2662 & -13.8338 & -9.7677 \\
U62 & 0.1327 & 0.2654 & -0.3946 & -19.6892 \\
U74 & 0.1320 & 0.2634 & -0.3760 & -4.6320 \\
\hline
\end{tabular}

TABLE 4: Results with $h=-0.5$ and $d=(1,1)$.

\begin{tabular}{lcccc}
\hline & $z_{1}^{(+)}$ & $z_{2}^{(+)}$ & $\mathrm{MR}_{11}^{(+)}$ & $\mathrm{MR}_{12}^{(+)}$ \\
\hline U4 & 0.1318 & 0.2635 & 19.6165 & 4.1730 \\
U6 & 0.1319 & 0.2639 & 29.576 & 2.6323 \\
U7 & 0.1163 & 0.2326 & 629.15 & 2.5548 \\
U13 & 0.1270 & 0.2559 & 1.9441 & 0.0082 \\
U17 & 0.1293 & 0.2587 & 0.0013 & 0.0027 \\
U20 & 0.1307 & 0.2614 & 5.3186 & 1.8572 \\
U38 & 0.1317 & 0.2633 & 40.2167 & 0.9733 \\
U39 & 0.1317 & 0.2635 & 13.8365 & 9.7731 \\
U62 & 0.1314 & 0.2627 & 0.3973 & 19.6946 \\
U74 & 0.1306 & 0.2613 & 0.3787 & 4.6374 \\
\hline
\end{tabular}

$\mathrm{MR}_{12}^{(+)}=0.0027$. In plant 7 , we confront a different case; 0.5 unit increment in the availability factor leads to a decrement of 1.2747 in $z_{1}$ and a huge decrement of 314.5723 in the failure rate. Now, consider $h=-0.5$ that the results are given in Table 4.

Again, for plant 13, if we decrease the availability factor from its current value 95.64 to $95.14(h=-0.5)$, with $d=$ $(1,1)$, the average time to repair and the failure rate are, respectively, decreased to 0.1270 and 0.2559 with $\mathrm{MR}_{11}^{(+)}=$ -1.9441 and $\mathrm{MR}_{12}^{(+)}=0.0082$.

An interesting point in this example is that we have run the procedure for different nonnegative $d$ and all directions lead to same results.

\section{Conclusions}

Marginal rates of substitution are one of the most frequently studied subjects in the content of nonparametric efficiency analysis. The problem of calculating marginal rates of substitution has been studied from different point of views. The existing trade-off analysis methods calculate the maximum changes in a specific throughput when another throughput is changed. In this case, a single measure of marginal rate is calculated. The current paper has shown how supporting surfaces of production technology in nonparametric techniques can be used to analyze the behavior of the firms. In this paper, we have shown that binding efficient supporting surfaces can be used to define different marginal rates of substitutions and in this sense, instead of getting a single measure of marginal rate of substitution as was done earlier in the literature, we can get different marginal rates of substitution to each frontier point. Numerical examples are used to illustrate the applicability of the proposed approach.

\section{Conflict of Interests}

The authors declare that there is no conflict of interests regarding the publication of this paper.

\section{Acknowledgment}

Financial support by Rasht Branch, Islamic Azad University Grant no. 4.5830, is gratefully acknowledged.

\section{References}

[1] A. Charnes, W. W. Cooper, and E. Rhodes, "Measuring the efficiency of decision making units," European Journal of Operational Research, vol. 2, no. 6, pp. 429-444, 1978.

[2] W. W. Cooper, L. M. Seiford, and J. Zhu, Handbook on Data Envelopment Analysis, Kluwer Academic Publishers, Norwell, Mass, USA, 2004.

[3] W. D. Cook and L. M. Seiford, "Data Envelopment Analysis (DEA) - thirty years on," European Journal of Operational Research, vol. 192, no. 1, pp. 1-17, 2009.

[4] Z. Huang, S. X. Li, and J. J. Rousseau, "Determining rates of change in data envelopment analysis," Journal of the Operational Research Society, vol. 48, no. 6, pp. 591-593, 1997.

[5] D. Rosen, C. Schaffnit, and J. C. Paradi, "Marginal rates and two dimensional level curve in DEA," Journal of Productivity Analysis, vol. 9, no. 3, pp. 205-232, 1998.

[6] W. W. Cooper, K. S. Park, and J. T. Pastor Ciurana, "Marginal rates and elasticities of substitution with additive models in DEA," Journal of Productivity Analysis, vol. 13, no. 2, pp. 105$123,2000$.

[7] V. E. Krivonozhko, O. B. Utkin, A. V. Volodin, I. A. Sablin, and M. Patrin, "Constructions of economic functions and calculations of marginal rates in DEA using parametric optimization methods," Journal of the Operational Research Society, vol. 55, no. 10, pp. 1049-1058, 2004.

[8] F. R. Førsund and L. Hjalmarsson, "Are all scales optimal in DEA? Theory and empirical evidence," Journal of Productivity Analysis, vol. 21, no. 1, pp. 25-48, 2004.

[9] F. R. Førsund, L. Hjalmarsson, V. E. Krivonozhko, and O. B. Utkin, "Calculation of scale elasticities in DEA models: direct and indirect approaches," Journal of Productivity Analysis, vol. 28, no. 1-2, pp. 45-56, 2007.

[10] B. M. Balk, R. Färe, and G. Karagiannis, "On directional scale elasticities," Journal of Productivity Analysis, vol. 43, no. 1, pp. 99-104, 2015.

[11] L. Khoshandam, R. K. Matin, and A. Amirteimoori, "Marginal rates of substitution in DEA with undesirable outputs: a directional approach," Measurement, vol. 68, pp. 49-57, 2015.

[12] M. Asmild, J. C. Paradi, and D. N. Reese, "Theoretical perspectives of trade-off analysis using DEA," Omega, vol. 34, no. 4, pp. 337-343, 2006. 
[13] R. D. Banker, A. Charnes, and W. W. Cooper, "Some models for estimating technical and scale inefficiencies in data envelopment analysis," Management Science, vol. 30, no. 9, pp. 10781092, 1984.

[14] A. Masoumzadeh, M. Toloo, and A. Amirteimoori, "Performance assessment in production systems without explicit inputs: an application to basketball players," IMA Journal of Management Mathematics, 2014.

[15] R. W. Shephard, Theory of Cost and Production Functions, Princeton University Press, Princeton, NJ, USA, 1970.

[16] A. Zanella, A. S. Camanho, and T. G. Dias, "Undesirable outputs and weighting schemes in composite indicators based on data envelopment analysis," European Journal of Operational Research, vol. 245, no. 2, pp. 517-530, 2015. 


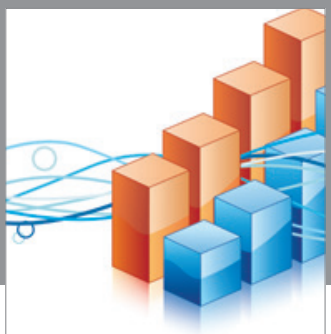

Advances in

Operations Research

vatem alat4

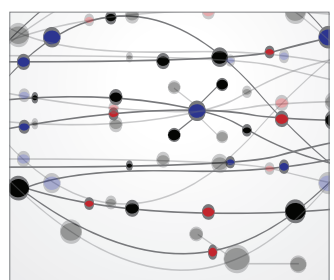

\section{The Scientific} World Journal
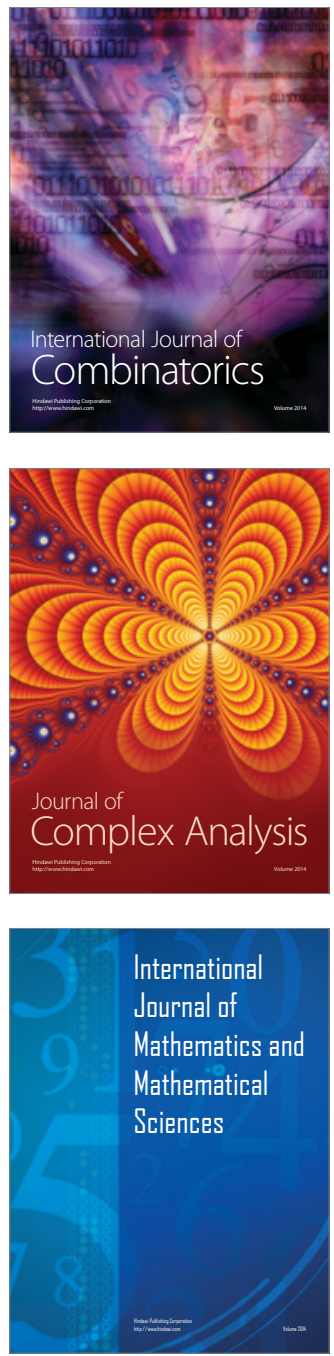
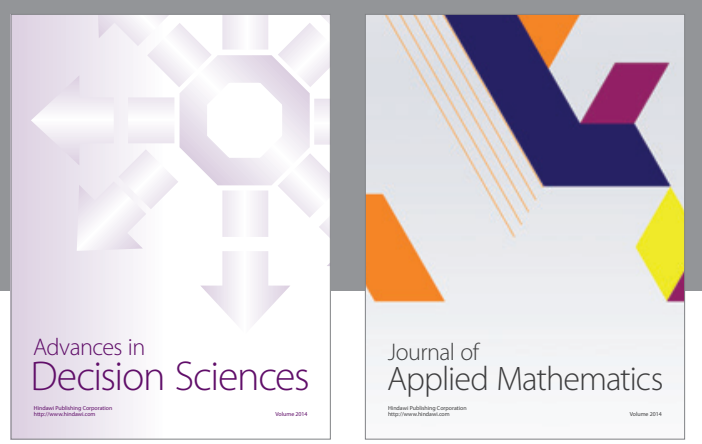

Algebra

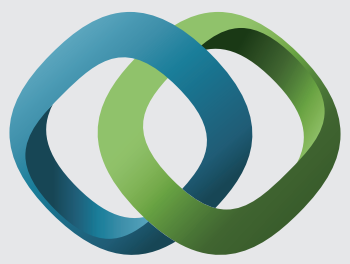

\section{Hindawi}

Submit your manuscripts at

http://www.hindawi.com
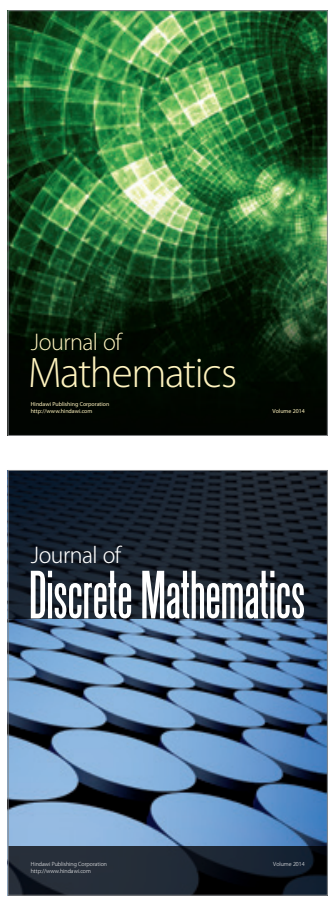

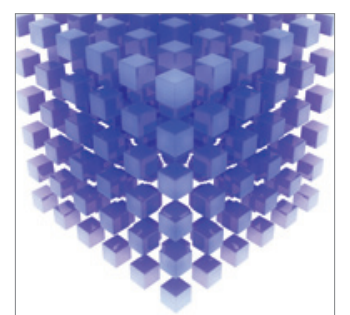

Mathematical Problems in Engineering
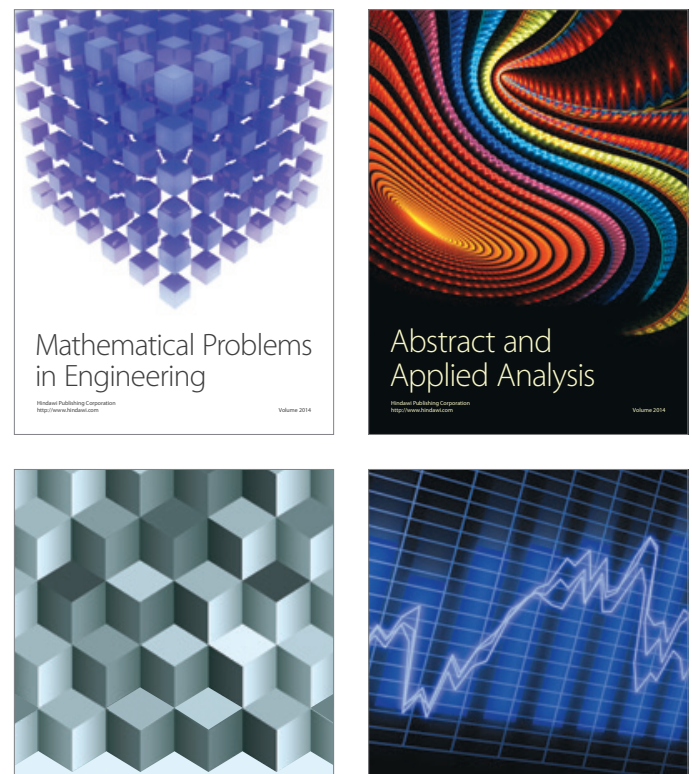

Journal of

Function Spaces

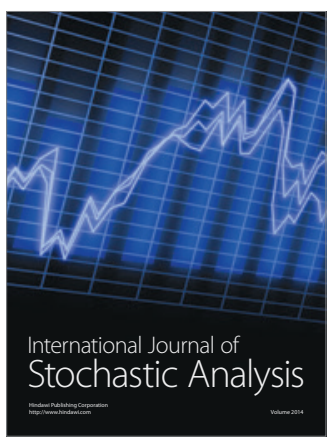

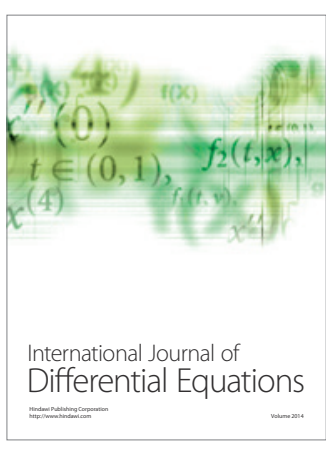
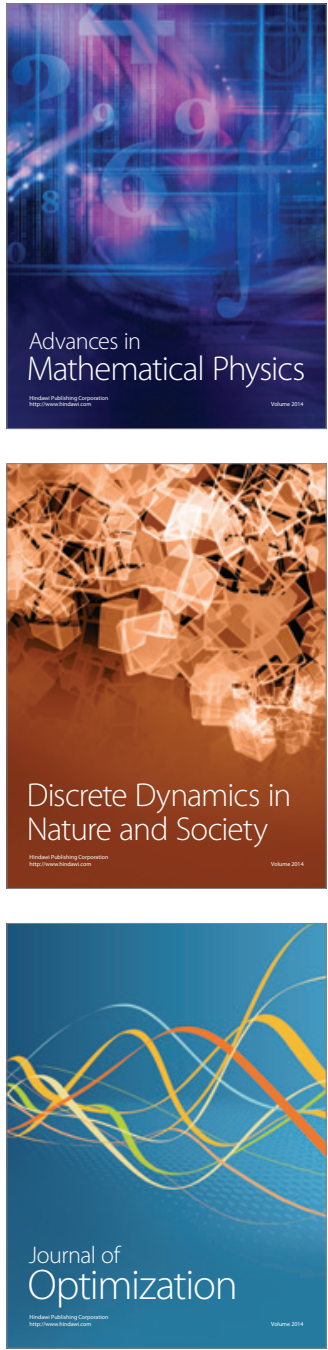